\title{
The comprehensive modernization of Ufa's electricity infrastructure
}

\author{
A. Y. Makarov ${ }^{1}$, Y. Hain ${ }^{2}$, I. Y. Bayramov ${ }^{1}$, V. Podshivalov ${ }^{2}$, \\ Y. A. Radygin ${ }^{1}$, E. Koifman ${ }^{2}$ \& I. S. Krutous ${ }^{1}$ \\ 'Joint Stock Company "Bashkirian Power Grid Company", Russia \\ ${ }^{2}$ Israel Electric Corporation Ltd., Israel
}

\begin{abstract}
The main challenges facing the electrical grid companies in the Russian Federation have been to increase its operational efficiency and improve the quality and reliability of its power supply.

In order to address these challenges, Bashkirian Power Grid Company (BPGC) together with Siemens LLC and the Israel Electric Corporation, Ltd. (IEC) conducted the analysis of global trends in the electricity distribution and infrastructure. Based on the results of the analysis, BPGC decided to consider an optimization and an upgrade of the Ufa grid infrastructure.

Siemens LLC, which is included in the approved reference list in electricity infrastructure projects, was engaged to perform the feasibility study.

Due to the technical complexity of the project, it was decided to hire the owner of one of the world's leading power grid engineering companies. IEC was chosen because of its commendable operational (electricity losses) records. Since 2010, IEC has deployed smart meters as a part of the smart grid project.

This paper describes a step-by-step approach to assessing the feasibility of Ufa's power grid modernization project.

Keywords: electricity distribution system, electricity losses, smart grid.
\end{abstract}

\section{Best practices of power grid infrastructure management}

Electric utilities must meet the increasing demand for reliable power distribution while coping with the decreasing tolerance for disruptions and outages. More than ever, power companies, forced to do more with less, recognize the need to improve the efficiency of their power transmission and distribution systems. 
Mostly, these changes are associated with the reforms that have been implemented in many countries in the energy sector - allocation of generation, transmission, distribution, and supply functions in separate companies, with generation and supply undergoing privatization and deregulation. Transmission and distribution sectors, usually remaining in fully-regulated areas, are frequently used by government structures as inflation-taming instruments. As a result, these sectors often experience a lack of funds for investment programs, while they are not exempt from the need to provide services determined by law. It should be noted that were funds available, implementation of projects do not always go according to schedule, as energy facilities, especially in cities and towns, suffer a constant restriction of building permits and licensing. As a result, transmission and distribution companies constantly search for solutions to improve the utilization of existing assets.

In addition to the reasons for the increasing requirements for reliability and quality of power supply described above, following the natural evolution of the industry in the last decade, we have witnessed revolutionary changes that are transforming the technological principles of the system, i.e. the impact of distributed generation. Mass penetration of distributed generation is connected primarily with the strategy of increasing the share of renewable energy in the overall energy balance. Electricity generation units based on solar, wind, and other natural resources, in spite of their current relatively modest contribution to the overall energy balance, have already demonstrated their impact on all sectors of the electricity industry. Considering the transmission and distribution sectors, the very paradigm of the electrical system is being challenged. We are witness to the transition from one-direction transmission of electricity of generation units to consumers to a system with reversing (two-directional) flows from distributed sources, often connected to the grid at medium and low voltage.

Many renewable sources are barely predictable for the purposes of dispatch control and require a high level of operational reserve, both in the generation and transmission sectors.

In these conditions the basic principles of system planning cease to be true and a new approach to meet the new conditions is needed.

Many areas of the existing electrical distribution systems can be improved through automation and new technological solutions. To manage the transmission and distribution systems in a new environment, the electricity system should be adapted to new working conditions, and primarily, the network topology and its transfer capacity.

Many companies use distribution solutions of the Distribution Management System (DMS) to monitor and manage the entire distribution system efficiently and reliably. This system provides the necessary support for dispatch centers. Improvement of the reliability and quality of the electricity supply, expressed in a decrease in the number of outages, and reduction of their duration, frequency, and voltage control, are the main goals of DMS implementation.

Implementation of DMS deployment occurs usually in stages and takes several years. For example, in the IEC, the project has been ongoing for more than seven years and will be finalized by the end of 2016 when all the necessary devices are 
assembled completely. The project began with the replacement of manually operated devices with remotely-operated ones. At this stage more than 2000 devices - circuit breakers, disconnectors, etc. have been replaced, and a remote-control system has been assembled. Currently, there are six regional control centers in the company equipped with modern equipment, data transfer via a SCADA system, and a central fully-automated dispatch center.

The next stage in the development of distribution systems associated usually with the implementation of the smart grid concept, commonly characterized in terms of the bidirectional flows of electricity and information and allows effective management of the distribution system, providing full integration of all system players. In technical terms, the smart grid is a set of transmission lines of all voltage levels, power conversion appliances, switchgear, protection and automation devices, information technology, and adapted control systems using high-speed data communication systems. The most important element is the smart grid management system, which monitors and controls the network element loads, and the quality and quantity of electricity supplied, based on the use of smart meters.

Installation of smart meters provides:

- Transition to a new level of electricity metering, accompanied by fundamental changes in the amount and availability of measured data (the most effective method to reduce commercial losses of electricity).

- A real opportunity to engage consumers in energy-saving processes and demand management.

- Acquisition of data for operation/supervisory control of the electricity system in real-time mode.

In the implementation of smart grid solutions and smart meters as part of these solutions, communication solutions play a very important role. Transferring data from the sensors/meters to the control center, and the commands/data in the opposite direction reliably and securely is a critical factor for the success of the smart grid. Therefore, dozens of pilot projects in the world have been realized and implemented in this direction. The IEC has been involved in joint ventures with the European Union and implements its own pilot and regular projects as of the beginning of this century:

- Project DLC + VIT4IP development and testing of high-speed narrowband

- communication lines based on IP.

- Project ENERsip to create an adaptive system for monitoring and control.

- Project FTTH (fiber-to-the-home): the communication system to the homeof the consumer is based on fiber optics.

- $\quad$ Project MICIE to evaluate real-time threats to critical infrastructure.

- $\quad$ Project DSM, demand-side management, by consumer demand control. 
- Transition of 6000 large consumers, responsible for $55 \%$ of electricity consumption in Israel to smart meters.

- Pilot project of households transition: 4,500 consumers, connected to smart meters of different technologies.

- Pilot project to analyze the impact of various tariff schemes on the behavior of households. The project is accompanied by the installation of 20,000 smart meters.

Implementation of smart grid projects (smart meters) should be based on the increased focus on cyber security, in all aspects.

Cyber-threat increase is caused by:

- $\quad$ The increased number of entry points and paths that can be exploited by potential offenders/hackers. Cyber security experts have already demonstrated that certain smart meters can be successfully attacked.

- The increased use of new systems and network technologies that can introduce new unknown risks.

- The interconnecting systems and networks that allow offenders wider access and the ability to spread dangerous activity; and,

- The increased amount of customer information being collected on systems provides monetary incentive for offenders to attack these systems.

The expert consensus is that the introduction of smart grids must be accompanied by significant reinforcement of cyber-defense systems. Thus, use of the world-leading practices in the power-grid field provides a high standard of power supply quality and reliability. The crucial factor is grid management process automation at all levels. Deploying smart meters plays an important role and provides the necessary data for a management system.

\section{Power-grid management in Russia and CIS}

Grid infrastructure conditions in Russia and CIS do not fully correspond to best international practices. A typical model of an approach to electricity grid management is described further on in this paper. This approach takes Ufa's electricity grid (belonging to Bashkirian Power Grid Company) as an example. However, it is applicable for other urban electricity grids in Russia and the CIS.

JSC BPGC, Bashkirian Power Grid Company, is the successor of Bashkirenergo in ownership and management of the power grid assets. Ufa, a large industrial city with a population of more than one million inhabitants, is the capital of the Republic of Bashkortostan, part of the Volga Federal District. Ufa's electricity supply is carried out by electricity networks of several voltage levels: $110-35-10-6-0.4 \mathrm{kV}$. Supply centers are $110 \mathrm{kV}$ or $35 \mathrm{kV}$ substations. The distribution network of 6-10 k V network is mainly a simple radial scheme, based on underground cables. Ufa's $6-10,0.4 \mathrm{kV}$ electricity grid management is geographically divided into seven regions. 
Operational monitoring of power parameters (power, currents, voltage levels) and of switch positions at substations are implemented with an operational information complex (OIK - Dispatcher) in real-time regime. The current level of remote control allows control and forecast of technological regimes for the 6-10 kV network at substations only (feeding centers). But the remote control and automation does not exist in $6-10 / 0.4 \mathrm{kV}$ transformation stations, affecting the quality of normal or maintenance regime management, leading to increased time for localization and liquidation of $6-10 / 0.4 \mathrm{kV}$ networks outages and, as a result, to increased unsupplied electricity.

Considering significant differences of the current power grid situation from the world's leading practices, BPGC management decided to estimate the effect of these differences on the operational results of company performance. BPGC technical specialists together with Siemens and IEC implemented a comparative analysis of the operational parameters of the Bashkirian grid company with those of the leading European companies operating electrical networks.

\section{Comparative analysis of operational indices for companies with different levels of power-grid infrastructure}

European companies in Spain, Belgium, Portugal, Italy, and Denmark were selected to compare the world's leading companies with the best practices in power-grid management. The leading companies from other regions, including IEC, were also listed for comparison.

The basic parameters used for the comparison were: supply of electric power, the number of disconnections, the outage duration in networks, and electricity loss, both technical and commercial.

\subsection{Electricity supply}

The annual electricity supply to BPGC consumers is about 18 TWh. (Fig. 1).

It is near the average value for the companies included in the survey, accordingly ensuring data comparability.

\subsection{Number and duration of electricity network outages}

The results of the analysis show that the average level of disconnected consumers of the companies included in the survey was 77.8 units (Fig. 2) and the average outage duration for a consumer was 114 minutes (Fig. 3) per $100 \mathrm{~km}$. of distribution electrical network. Similar figures in the Bashkirían Power Grid Company were 373.3 units (level of disconnected consumers) and 229 minutes (outage duration per consumer) showing the need for improvement of BPGS's automation level.

\subsection{Electricity energy losses by region}

As part of the analysis, the average level of electricity losses has been compared in different countries as an important indicator of the power grid infrastructure status [1]. This parameter is in a range from $4 \%$ in South Korea and Israel to $25 \%$ in India (Fig. 3). 
564 Energy Production and Management in the 21st Century, Vol. 1

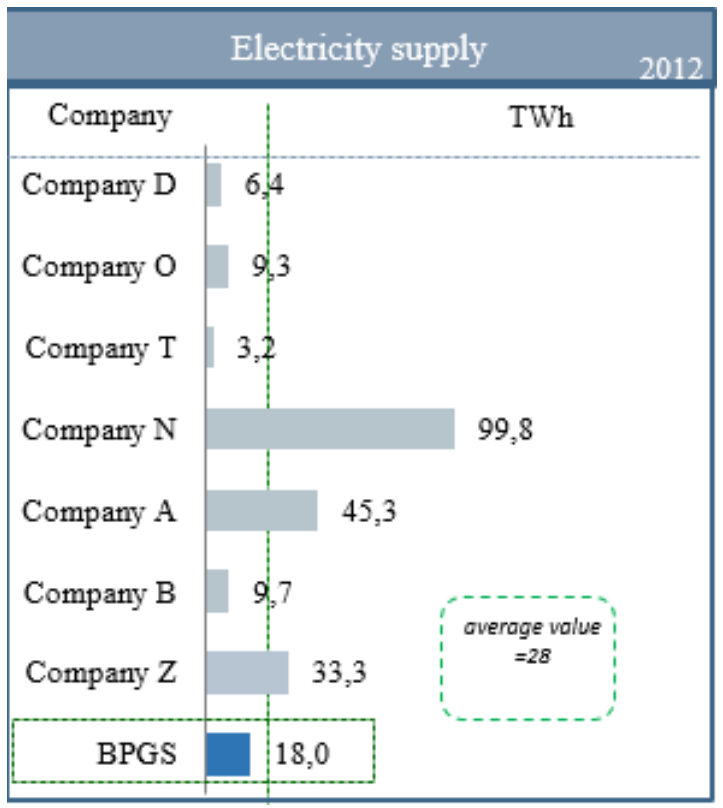

Figure 1: $\quad$ Electricity support.

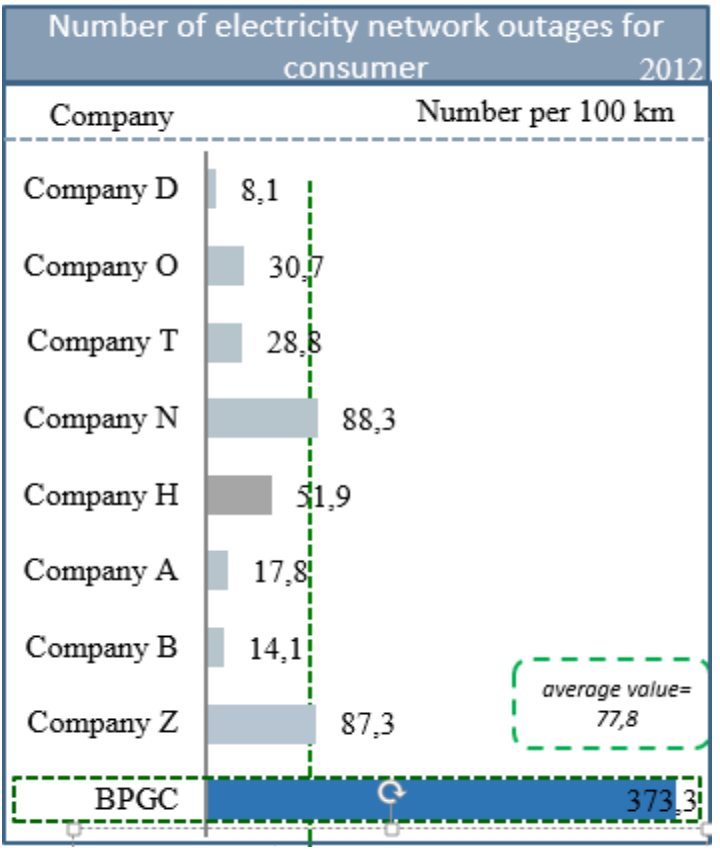

Figure 2: Number of electricity network outages. 


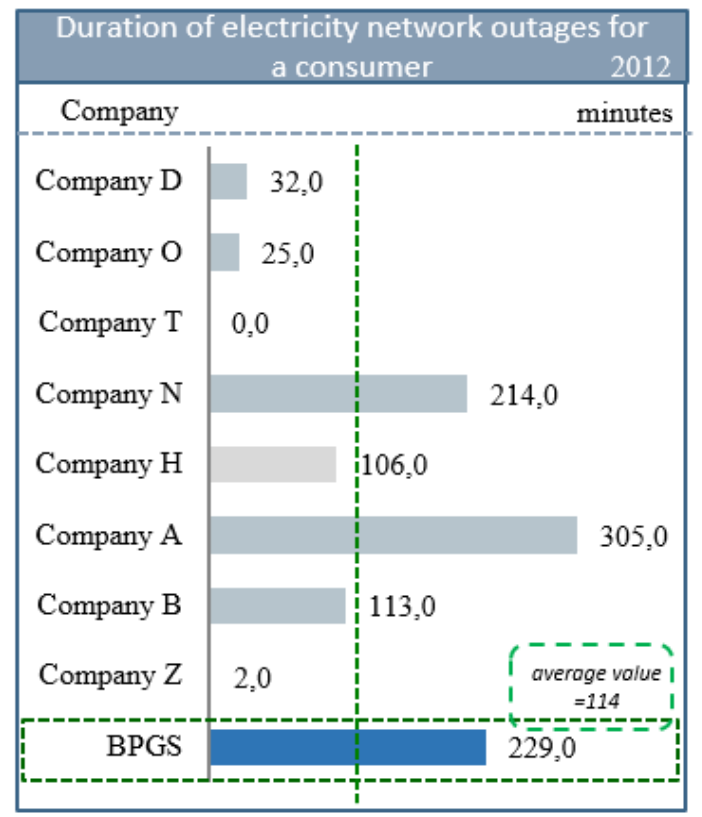

Figure 3: Duration of electricity network outages.

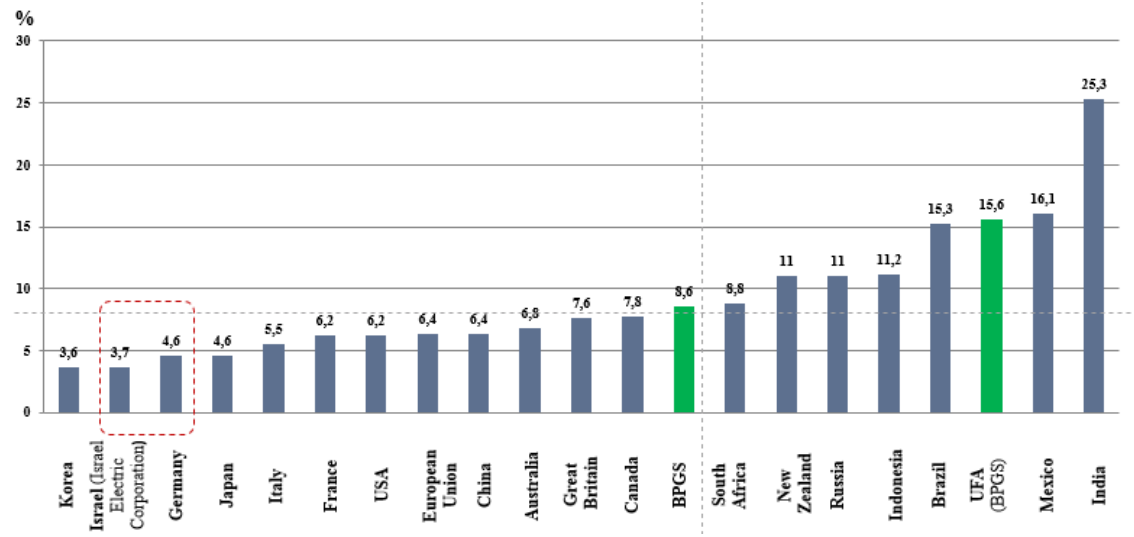

Figure 4: $\quad$ Energy losses.

The electricity energy losses in Ufa are at $15.6 \%$, suggesting a significant potential for reduction.

Benchmarking results show a significant gap between the operating efficiency of power grid companies in Russia and the CIS and industry world leaders.

To assess the possible effects of the world's best management practices and their introduction to Ufa's electricity grid, BPGC management decided to prepare a feasibility study for a grid infrastructure modernization project with introduction to smart grid technology elements. 


\section{Organizational framework of feasibility study preparation}

In order to ensure feasibility study quality, it was important to choose an executor with sufficient experience in similar work implementation. In view of the required expertise, Siemens, an internationally recognized leader in the field of electrical smart grids, was commissioned as an executor of this study.

Due to the lack of experience with smart grid technologies by BPGC and to mitigate the risks of a deficient feasibility report, an independent consultant was engaged. IEC, with extensive experience in modern electric equipment operation and with one of the lowest losses level in the industry, was chosen as the consultant. Since 2010, the company has deployed smart meters and executed other projects in the smart grids' field. IEC's expertise was sufficient proof that the company had the ability to make well-grounded recommendations regarding the development of Ufa's grid infrastructure.

JSC BPGC became the first Russian (CIS) company to contract with leading global partners to jointly assess the feasibility of a comprehensive grid modernization project for a large city.

The next step, after choosing the executor and the technical consultant, was to formulate the precise objectives of the feasibility study. This was achieved by preparing, with JSC BPGC, Siemens, and the IEC, a feasibility study specification for modernizing the Ufa power grid.

The work structure as defined within the feasibility study is represented as follows:

1. The Ufa power grid data collection;

2. Analysis of data quality;

3. Modeling of the electric Ufa "as is" network;

4. The network current state analysis based on computer simulation results;

5. Preparation of different scenarios for a perspective development plan for 2020, including smart grid elements for each scenario;

6. Cost-benefit analysis for each scenario and selection of the preferred one;

7. Plan preparation for the transition from the current situation to the power grid target model.

\section{The Ufa power grid infrastructure development - feasibility study approach}

The approach to the feasibility study preparation for the grid infrastructure modernization project of a large city, described below, was formed by JSC BPGC, Siemens, and the IEC and was used to assess the feasibility of development scenarios for the Ufa electricity grid.

The most time-consuming step of the feasibility study was collecting and analyzing electricity system data for the development of the "as is" network model. Technical data relating to the all existing equipment for voltage level $6 \mathrm{kV}$ and above, including the corresponding loads, was used for the computer simulations. The data was structured by completing special 
questionnaires. In the event of no computerized database containing all the necessary information, the data for modelling was completed in the questionnaires manually. The survey forms took several months to complete as this was performed for an electricity system of a large city with considerable quantity of lines, generation units, transformers, and other electrical devices.

Preliminary adequacy analysis of the collected data was executed by comparing model samples with the single-line diagram of the existing urban network and/or checking node by node the data and lines for the network model and the real network.

Simultaneously, with the preliminary data analysis, "as is" network modeling was implemented. During preparation, the model was compared with the existing single-line diagram. In the event of a discrepancy, the model was corrected to match the real network while errors and inaccuracies in the source data were identified. Wherever necessary, the collected data was corrected. Therefore, the model-building process was iterative. Together with the visual inspection of the modelled topology, load comparison between computer model results and the control measurements in the existing grid (maximum winter, minimum summer) was performed. Measured values were provided for each node on the questionnaire-completing stage.

When the full identity of network topology between the model and the singleline diagram of the real network was achieved, and node loads similarity was found to be satisfactory (calculated loads corresponded to those indicated in the raw data readings of control measurements) a "model behavior test" was performed. In the test, "standard disturbancy" was injected into the urban network model (in this case, the behavior of the real network in a similar situation should be known to the client company). The "as is" network model was considered to be complete only after successful verification of model generated regimes.

Following the completion of the electrical "as is" network model, an assessment of the current state of the network and identification of existing system bottlenecks was executed. Load density, equipment amortization, protection and automation system, short-circuit current level, voltage stability, technical losses; reliability and other factors were considered during this phase. The final conclusions regarding the status of the existing urban electricity grid was based not only on the current data, but also the projected loads. Network analysis considered future development, required implementation preparation of network load changes (connected loads) forecast for the next five years, at least. This forecast at the medium voltage level was required for each urban district.

Several alternative scenarios of grid development were formed after completion of the "as is" network analysis. Under these scenarios, various levels of network modernization and automation were simulated to improve the grid's current performance.

A smart grid concept was developed for each scenario, including grid management system, metering, and control systems. Possible solutions for absorption of distributed generation units was also considered during the concept development. 
Inclusion of smart grid components was considered while forming a network management system: collection and processing of information related to the status and loads of network elements; a warning system of the pre-fault and fault conditions in the network; accident data processing; search and localization of damaged network sections, and recovery of the power supply via the undamaged parts of the network after the accident localization, and automation of the dispatching functions.

Smart grid equipment was installed in the metering and control system. The equipment included: devices and software for the automation of transmitted and supplied electric metering; electric energy $(\mathrm{kWh})$ and power $(\mathrm{kW}, \mathrm{MW})$ balance preparation; loads assessment (A) at different voltage levels; and load curves preparation and processing. Recommendations were suggested regarding the integration of the new load into an existing network.

The smart grid concept regarding distributed/renewable generation units will be included in each scenario: devices and software for collecting and processing information regarding the operation of distributed generation units; recommendations for operational-dispatching personnel. Moreover, operational-dispatching personnel will receive recommendations for network optimization. These recommendations will consider the operation of distributed generation units and the impact of connection on the existing network.

Careful attention should be placed on system cyber security while developing the smart grid concept.

As mentioned above, the main purpose of network modernization is to improve the quality and reliability of the power supply. But, if the power grid project had been executed by a private investor-owned company, there would have been an additional requirement for cost-effectiveness (profitability) of the project. Since these requirements were sometimes contradictory, clearly formulated criteria for scenarios comparison was required from the grid company. This was a pre-condition for the study executor to develop future grid scenarios complying with the client's business strategy. Some examples of possible criteria are: power supply reliability, electricity supply quality, technical losses, level of commercial losses, operating costs, and the investments required. The number of criteria and their combination used in each particular project, depended on the customer's business targets.

Upon approval of the compared criteria, the feasibility study performer prepared a network model and developed a smart grid concept for each scenario. Furthermore, the study executor estimated the effectiveness of the activities proposed for each scenario (implementing cost-benefit analysis) and compared scenarios with previously agreed criteria.

After the best-case scenario was selected, the study executor created a detailed project plan for the transition from the current situation to the target model, including the establishment of a project schedule and preparation of budget estimation. Based on this plan, the power grid company approved the investment program and proceeded to the stage of implementation of the comprehensive urban electricity grid modernization project. 


\section{Conclusions}

This paper describes the best world practices for power grid management, and emphazises the gaps in operational efficiency between world leading companies and grid companies in Russia and CIS. The reasons for consideration of urban grid modernization projects in Russia and the CIS are explained.

The paper also outlines the approach to a feasibility study preparation for a large urban-grid development project, developed by Siemens, Bashkirian power grid company, and the IEC and implemented in the city of Ufa. The described approach is applicable, with minor modifications, for assessing the feasibility of grid modernization for any large and medium city in Russia and the CIS.

\section{Reference}

[1] Paper of the World Energy Council "Smart Grids: Best Practice Fundamentals for a Modern Energy System”, p. 6, 2012, London, www.worldenergy.org 